MATHEMATICS OF COMPUTATION

Volume 74, Number 252, Pages 1967-1982

S 0025-5718(05)01746-1

Article electronically published on April 15, 2005

\title{
DEFORMATIONS OF MAASS FORMS
}

\author{
D. W. FARMER AND S. LEMURELL
}

\begin{abstract}
We describe numerical calculations which examine the PhillipsSarnak conjecture concerning the disappearance of cusp forms on a noncompact finite volume Riemann surface $S$ under deformation of the surface. Our calculations indicate that if the Teichmüller space of $S$ is not trivial, then each cusp form has a set of deformations under which either the cusp form remains a cusp form or else it dissolves into a resonance whose constant term is uniformly a factor of $10^{8}$ smaller than a typical Fourier coefficient of the form. We give explicit examples of those deformations in several cases.
\end{abstract}

\section{INTRODUCTION AND STATEMENT OF RESULTS}

We summarize the basic facts about eigenvalues of the Laplacian on compact and noncompact surfaces, and then we describe our calculations.

1.1. Weyl's law. The Laplacian $\Delta$ on a compact Riemann surface $S$ has a discrete spectrum $0=\lambda_{0}<\lambda_{1} \leq \lambda_{2} \cdots$. As is traditional, we write $\lambda_{j}=\frac{1}{4}+i t_{j}^{2}=\frac{1}{4}+R_{j}^{2}$, and we occasionally refer to $R_{j}$ as the "eigenvalue". There is a precise estimate for the magnitude of $\lambda_{n}$ given by Weyl's law:

$$
N(T):=\#\left\{\left|t_{n}\right| \leq T\right\} \sim \frac{V}{4 \pi} T^{2},
$$

where $V=\operatorname{Vol}(S)$ and eigenvalues are repeated according to their multiplicity.

1.2. Noncompact surfaces. If $S$ is noncompact but has finite volume, then the situation is more subtle, for $\Delta$ will have both a discrete and a continuous spectrum. See $\mathrm{Iw}$ for a complete discussion. The continuous spectrum consists of the interval $\left[\frac{1}{4}, \infty\right)$, and for $s \in\left[\frac{1}{4}, \infty\right)$ we write $s=\frac{1}{4}+i t^{2}$. The eigenfunctions in the continuous spectrum are given by Eisenstein series, and the eigenfunctions for the discrete spectrum are called Maass forms or nonholomorphic cusp forms. Here Weyl's law states

$$
M(T)+N(T) \sim \frac{V}{4 \pi} T^{2}
$$

Received by the editor February 19, 2003 and, in revised form, April 30, 2004.

2000 Mathematics Subject Classification. Primary 11F03; Secondary 11F30.

Key words and phrases. Maass forms, deformations, Phillips-Sarnak conjecture, Teichmuller space.

Research of the first author was supported in part by the National Science Foundation and the American Institute of Mathematics.

Research of the second author was supported in part by "Stiftelsen för internationalisering av högre utbildning och forskning" (STINT).

(C)2005 American Mathematical Society Reverts to public domain 28 years from publication 
where $M(T)$ is the contribution of the continuous spectrum, given by

$$
M(T)=\frac{1}{4 \pi} \int_{-T}^{T}-\frac{\varphi^{\prime}}{\varphi}\left(\frac{1}{2}+i t\right) d t,
$$

where $\varphi$ is the determinant of the scattering matrix for the Eisenstein series.

It is a fundamental problem to determine which of $M(T)$ or $N(T)$ makes the main contribution to the spectrum. If $S$ is a surface corresponding to a congruence subgroup, then techniques from analytic number theory can be used to show that $M(T) \ll T \log T ;$ so then

$$
N(T) \sim \frac{V}{4 \pi} T^{2}
$$

as in the compact case. This is also conjectured to hold for any arithmetic surface [S1, S2] (see Ta for a discussion of arithmetic Fuchsian groups). For arithmetic surfaces we also have the deep conjecture of Selberg that $\lambda_{1} \geq \frac{1}{4}$.

1.3. Nonarithmetic surfaces and the destruction of cusp forms. If $S$ is a noncompact nonarithmetic finite volume surface, then work of Colin de Verdiere [C1, C2 and Phillips and Sarnak [PS1, PS2, PS3 suggests that $S$ should in general have no discrete spectrum. Thus, for noncompact surfaces Maass forms are rare and unusual and are mostly confined to arithmetic surfaces.

The nonexistence of cusp forms on generic noncompact surfaces is commonly stated in terms of the "destruction" of cusp forms. Namely, if $S$ has a Maass form with eigenvalue $\lambda$, then for almost all small deformations of $S$, the new surface will have no discrete eigenvalues in a neighborhood of $\lambda$. That is, almost all deformations destroy the cusp form. In the case of variable negative curvature [C1, C2 the deformation space is infinite dimensional and a generic perturbation of the metric will destroy the cusp form. In the constant negative curvature case [PS1, [PS2], PS3 deformations are given by the Teichmüller space of the surface, which is finite dimensional. This situation is much more subtle.

As described by Phillips and Sarnak, under deformations of the surface the elements of the discrete spectrum tend to become resonances, that is, poles of the scattering matrix of the Eisenstein series. In the case of a group with one cusp, the Eisenstein series satisfies a functional equation of the form $E(z ; s)=\varphi(s) E(z ; 1-s)$, where $\varphi(s) \varphi(1-s)=1$. A cusp form is destroyed if its eigenvalue moves off the line $\Re(s)=\frac{1}{2}$ to become a pole of $\varphi(s)$ in $\Re(s)<\frac{1}{2}$. See also [L, [P], W].

Note that by the expression for $M(T)$ in terms of $\varphi(s)$ above, this process does not change Weyl's law, although it does change the balance between $M(T)$ and $N(T)$.

The pole of $\varphi(s)$ in $\Re(s)<\frac{1}{2}$ corresponds to a zero of $\varphi(s)$ in $\Re(s)>\frac{1}{2}$. Avelin [A] has directly verified the destruction of cusp forms by tracking such zeros. In this paper we take a complementary approach and directly track the cusp forms along deformations which do not destroy the form. It would be interesting to compare our calculations with the results of Avelin.

1.4. The results of our calculations. In this paper we describe numerical calculations on noncompact surfaces $S$ which indicate that while it is true that almost all deformations destroy any given cusp form, individual cusp forms have deformations along which they are not destroyed. Our calculations indicate that, if $S$ has a nontrivial Teichmüller space, then for each Maass form on $S$ there is a 
continuous family of Teichmüller deformations on which the (deformations of the) Maass form lives. We explicitly describe these deformations in some simple cases.

A summary of our observations is as follows.

(1) Suppose $S_{0}$ has one cusp, the Teichmüller space of $S_{0}$ has dimension $d$, and $f_{0}$ is a Maass form on $S_{0}$ with eigenvalue $\lambda_{0}$. Then there is a continuous $(d-1)$ parameter family of deformations $S(t)$, Maass forms $f(t)$, and a function $\lambda(t)$, such that $S(0)=S_{0}, f(0)=f_{0}$, and $\lambda(0)=\lambda_{0}$.

In the above situation we say that " $f$ lives on a $(d-1)$-parameter family of deformations of $S^{\prime \prime}$. Also, if $f$ and $g$ are Maass forms on surfaces $S_{1}$ and $S_{2}$, respectively, then we say that $f$ and $g$ are equivalent if there exists a continuous family of deformations which sends $S_{1}$ to $S_{2}$ and sends $f$ to $g$.

Note that these calculations are reasonable in terms of the Phillips-Sarnak phenomenon. The condition that the eigenvalue remain on the line $\Re(s)=\frac{1}{2}$ imposes one condition on the deformation, and that condition should be satisfied on a codimension 1 subset of Teichmüller space. Furthermore, that set should be a real analytic subvariety.

(2) There exist Maass forms $f$ on a surface $S$ with 2-dimensional Teichmüller space, such that $f$ lives on two independent 1-parameter families of deformations of $S$.

(3) There exist Maass forms which are not equivalent to a Maass form on any arithmetic surface.

Since the definition of cusp form requires invariance under a group and vanishing constant term in the Fourier expansion, neither of those conditions can be verified by a floating point numerical calculation. In particular, the functions which we claim are cusp forms may not actually be invariant under the group, and more critically to our purpose here, they may not actually vanish at the cusp. As we discuss in Section 4 (see particularly Section 4.1.1), our calculations are done to a precision of one part in $10^{8}$, so there is no way for us to rule out the possibility that our "cusp forms" actually have a nonzero constant term which is a factor of $10^{8}$ smaller than the other Fourier coefficients. Throughout the paper we speak as if we are finding cusp forms, but it is possible that we are actually finding 1-parameter families of noncusp forms having unusually small constant Fourier coefficient.

In the next section we give basic definitions and describe our calculations. In the following section we describe two families of Riemann surfaces for which we explicitly found deformations which do not destroy a cusp form. In the final section we describe our calculations.

\section{DEFINITIONS AND DESCRIPTION OF CALCULATIONS}

We summarize standard facts about the relationship between Riemann surfaces and subgroups of $\operatorname{PSL}(2, \mathbb{R})$, and we describe the surfaces on which we performed our calculations.

2.1. Subgroups of $P S L(2, \mathbb{R})$ and $\mathcal{H} / \Gamma$. The group $P S L(2, \mathbb{R})$ acts on the upper half-plane $\mathcal{H}=\{z=x+i y: x, y \in \mathbb{R}, y>0\}$ by linear fractional transformations:

$$
\left(\begin{array}{ll}
a & b \\
c & d
\end{array}\right)(z)=\frac{a z+b}{c z+d}
$$

Here $\mathcal{H}$ is equipped with the hyperbolic metric $d s^{2}=y^{-2}\left(d x^{2}+d y^{2}\right)$ and area element $d A=y^{-2} d x d y$. If $\Gamma \subset P S L(2, \mathbb{R})$ is a discrete subgroup, then $\mathcal{H} / \Gamma$ is 
a Riemann surface of constant negative curvature -1 . Throughout the paper we consider the case where $\mathcal{H} / \Gamma$ is noncompact and has finite area, and in this case we say that $\Gamma$ is a cofinite subgroup of $P S L(2, \mathbb{R})$. The surface $\mathcal{H} / \Gamma$ can be compactified by the addition of a finite set of points. These missing points are referred to as "cusps", and they correspond to (conjugacy classes of) parabolic subgroups of $\Gamma$.

We use the standard notation for the signature of Riemann surface $S$ :

$$
\operatorname{sig}(S)=\left\{g,\left\{m_{1}, \ldots, m_{k}\right\}, \nu\right\},
$$

where $g$ is the genus of $S, m_{1}, \ldots, m_{k}$ are the orders of the elliptic points of $S$, and $\nu$ is the number of cusps of $S$. In the above notation, the Teichmüller space of $S$ has (real) dimension $6 g-6+2 k+2 \nu$.

If $\operatorname{sig}(S)$ is as given above, then $S=\mathcal{H} / G$ where $G \subset P S L(2, \mathbb{R})$ has presentation $G=\left\langle a_{1}, b_{1}, \ldots, a_{g}, b_{g}, e_{1}, \ldots, e_{k}, p_{1}, \ldots, p_{\nu} \mid e_{j}^{m_{j}}=1,\left[a_{1}, b_{1}\right] \cdots\left[a_{k}, b_{k}\right] e_{1} \cdots e_{k} p_{1} \cdots p_{\nu}=1\right\rangle$.

The equivalence between Riemann surfaces $S$ and groups $\Gamma \subset P S L(2, \mathbb{R})$ allows us to phrase everything in terms of the group. This is useful for computer calculations because we can represent the group by a convenient set of generators.

2.2. Maass forms and Hecke congruence subgroups. A Maass form on a group $\Gamma \subset P S L(2, \mathbb{R})$ is a function $f: \mathcal{H} \rightarrow \mathbb{R}$ which satisfies

(1.1) $f(\gamma z)=f(z)$ for all $\gamma \in \Gamma$,

(1.2) $f$ vanishes at the cusps of $\Gamma$, and

(1.3) $\Delta f=\lambda f$ for some $\lambda>0$, where

$$
\Delta=-y^{2}\left(\frac{\partial^{2}}{\partial x^{2}}+\frac{\partial^{2}}{\partial y^{2}}\right)
$$

is the Laplace-Beltrami operator on $\mathcal{H}$. Note that Maass forms on $\Gamma$ are elements of the discrete spectrum of $\Delta$ on $S=\mathcal{H} / \Gamma$.

If $\Gamma$ is cofinite, then it contains a parabolic element, which we can conjugate to $T=\left(\begin{array}{ll}1 & 1 \\ 0 & 1\end{array}\right)$. Thus, we may assume $f(z)=f(z+1)$. By (1.3) we find that $f(z)$ has a Fourier expansion of the form

$$
f(z)=\sqrt{y} \sum_{n \neq 0} a_{n} K_{i R}(2 \pi|n| y) \exp (2 \pi i n x),
$$

where $K_{\nu}(t)$ is a Bessel function and $\lambda=\frac{1}{4}+R^{2}$.

Maass forms naturally arise in number theory $[\mathrm{Iw}]$ in the case of $\Gamma=\Gamma_{0}(q)$. Here $\Gamma_{0}(q)$ is the Hecke congruence group

$$
\Gamma_{0}(q)=\left\{\left(\begin{array}{ll}
a & b \\
c & d
\end{array}\right) \in \operatorname{PSL}(2, \mathbb{Z}): q \mid c\right\} .
$$

It is usually more convenient to consider a slightly larger group $\Gamma_{0}^{*}(q)$, defined as follows. Suppose $t \mid q$ and $(t, q / t)=1$, and choose $a, b$ so that $a t-b n / t=1$. Let

$$
H_{t}(q)=\left(\begin{array}{cc}
a t & b \\
q & t
\end{array}\right)
$$

and note that $H_{t}(q)$ normalizes $\Gamma_{0}(q)$, the $H_{t}(q)$ are defined up to multiplication by an element of $\Gamma_{0}(q)$, and $H_{t}(q)^{2} \in \Gamma_{0}(q)$. The $H_{t}(q)$ are called the Fricke involutions for $\Gamma_{0}(q)$. If $q$ is squarefree, then we let $\Gamma_{0}^{*}(q)$ be the group generated by $\Gamma_{0}(q)$ and all of the $H_{t}(q)$ for $t \mid q$. This is a maximal discrete subgroup of $\operatorname{PSL}(2, \mathbb{R})$. If $q$ is not squarefree, then the group generated by $\Gamma_{0}(q)$ and all of the $H_{t}(q)$ for $t \| q$ may 
TABLE 2.1 .

\begin{tabular}{|r|l|l|}
\hline \multicolumn{1}{|c|}{$\operatorname{sig}\left(S_{0}(q)\right)$} & $\operatorname{sig}\left(S_{0}^{*}(q)\right)$ \\
\hline 5 & $\{0,\{2,2\}, 2\}$ & $\{0,\{2,2,2\}, 1\}$ \\
\hline 6 & $\{0,\{\}, 4\}$ & $\{0,\{2,2,2\}, 1\}$ \\
\hline 7 & $\{0,\{3,3\}, 2\}$ & $\{0,\{2,2,3\}, 1\}$ \\
\hline 8 & $\{0,\{\}, 4\}$ & $\{0,\{2,2,2\}, 1\}$ \\
\hline 9 & $\{0,\{\}, 4\}$ & $\{0,\{2,2,2\}, 1\}$ \\
\hline 10 & $\{0,\{2,2\}, 4\}$ & $\{0,\{2,2,4\}, 1\}$ \\
\hline 11 & $\{1,\{\}, 2\}$ & $\{0,\{2,2,2,2\}, 1\}$ \\
\hline
\end{tabular}

or may not be a maximal discrete group. If it is, then we call it $\Gamma_{0}^{*}(q)$. If it is not, then we adjoin as many more involutions as possible. See below for examples with $q=8$ and 9 . We write $S_{0}(q)=\mathcal{H} / \Gamma_{0}(q)$ and $S_{0}^{*}(q)=\mathcal{H} / \Gamma_{0}^{*}(q)$. See Co for an interesting discussion of the relationship between $S_{0}(q)$ and $S_{0}^{*}(q)$.

The groups $\Gamma_{0}^{*}(q)$ are more convenient for our purposes because $\Gamma_{0}^{*}(q)$ has fewer cusps than $\Gamma_{0}(q)$, and this simplifies the search for cusp forms. In fact, all of the cases we consider here have just one cusp. Since the Teichmüller spaces of $\Gamma_{0}(q)$ and $\Gamma_{0}^{*}(q)$ are essentially the same, there is no loss in considering the larger group.

If $q=1,2,3$, or 4 , then $\Gamma_{0}^{*}(q)$ is conjugate to a Hecke triangle group. These groups cannot be deformed. Maass forms on Hecke triangle groups have been extensively studied by Hejhal [H1], [H2], [H3]. If $q \geq 5$, then $\Gamma_{0}(q)$ can be deformed; we concentrate on the cases $q=5,6,8,9$, and 11 .

Table 2.1 gives the signature of $S_{0}(q)$ and $S_{0}^{*}(q)$ for small $q$.

Note that $S_{0}^{*}(5), S_{0}^{*}(6), S_{0}^{*}(8)$, and $S_{0}^{*}(9)$ have the same signature, so these surfaces are deformations of each other. That family of surfaces, along with deformations of $S_{0}^{*}(11)$, serve as our examples in this paper. In the next section we give explicit deformations of these surfaces.

\section{The Deformations}

We explicitly describe deformations of the surfaces $S_{0}^{*}(q)$ for $q=5,6,8,9$, and 11 .

3.1. Generators. Since these surfaces have genus 0 and one cusp, they can be realized from groups generated by elliptic matrices along with the single parabolic matrix $T=\left(\begin{array}{ll}1 & 1 \\ 0 & 1\end{array}\right)$. Let $r_{k}(x, y)$ be the matrix which acts as a rotation by $2 \pi / k$ around the point $x+i y \in \mathcal{H}$ :

$$
r_{k}(x, y)=\left(\begin{array}{cc}
c y-s x & s\left(y^{2}+x^{2}\right) \\
-s & c y+s x
\end{array}\right),
$$

where $c=\cos (\pi / k)$ and $s=\sin (\pi / k)$.

We will give an explicit description of our groups in terms of $T$ and $r_{k}(x, y)$. First we describe various groups containing free parameters, and then we indicate how they specialize to various $\Gamma_{0}^{*}(q)$. 

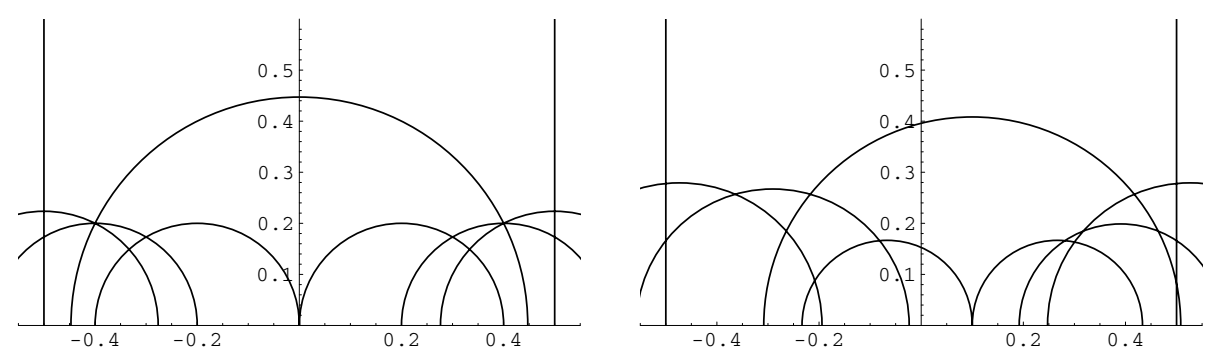

Figure 3.1. The region above the arcs and between the lines is a fundamental domain for $\Gamma_{2,2,2}(a, b)$ for $(a, b)=(5,0)$ and $(a, b)=$ $\left(6, \frac{1}{10}\right)$, respectively.

3.2. Signature $\{0,\{2,2,2\}, 1\}$. Let $\Gamma_{2,2,2}(a, b)=\left\langle T, g_{1}, g_{2}, g_{3}\right\rangle$ with

$$
g_{1}=r_{2}(b, 1 / \sqrt{a}), \quad g_{2}=r_{2}(x, \sqrt{y}), \quad g_{3}=T g_{1} g_{2},
$$

where

$$
\begin{aligned}
x & =\frac{1}{2}\left(\frac{2}{a}+b+Y\right), \\
y & =\frac{1}{2}\left(-\frac{4}{a^{2}}+\left(1-\frac{2}{a}+b\right)(-b+Y)\right), \\
Y & =\sqrt{\frac{4}{a^{2}}+b^{2}} .
\end{aligned}
$$

One can check that $\Gamma_{2,2,2}$ is well defined for $(a, b)$ in a neighborhood of $\{(a, 0) \mid a>$ $4+\varepsilon\}$ and that $\Gamma_{2,2,2}(a, b)$ satisfies the relations $g_{1}^{2}=g_{1}^{2}=g_{3}^{2}=1$ and $g_{1} g_{2} g_{3} T=1$. Thus, $\mathcal{H} / \Gamma_{2,2,2}(a, b)$ has signature $\{0,\{2,2,2\}, 1\}$.

Figure 3.1 shows the action of the generators of $\Gamma_{2,2,2}(a, b)$ on the upper halfplane, along with some additional elements of the group. Note that the figures have left/right symmetry when $b=0$.

In the figure on the left, the region above the four smallest circles is a fundamental domain for $\Gamma_{0}(5)$, and the region above the three largest circles is a fundamental domain for $\Gamma_{0}^{*}(5)$.

3.3. Signature $\{0,\{2,2,2,2\}, 1\}$. Let $\Gamma_{2,2,2,2}(a, b, c, d)=\left\langle T, g_{1}, g_{2}, g_{3}, g_{4}\right\rangle$, with

$$
g_{1}=r_{2}(a, x), \quad g_{2}=r_{2}(b, y), \quad g_{3}=r_{2}(c, z), \quad g_{4}=r_{2}(1 / 2, d),
$$

where

$$
\begin{aligned}
& x=\sqrt{\frac{(a-b)\left(\left(\frac{1}{2}+a\right)\left(-\frac{1}{2}+c\right)+d^{2}\right)}{\frac{1}{2}-c},} \\
& y=d \sqrt{\frac{(a-b)(b-c)}{\left(\frac{1}{2}+a\right)\left(\frac{1}{2}-c\right)}}, \\
& z=\sqrt{\frac{(b-c)\left(\left(\frac{1}{2}+a\right)\left(-\frac{1}{2}+c\right)+d^{2}\right)}{\frac{1}{2}+a}} .
\end{aligned}
$$


One can check that $\Gamma_{2,2,2,2}$ is well defined in a neighborhood $(-1 / 3,0,1 / 3$, $1 /(2 \sqrt{11}))$ and that $g_{1} g_{2} g_{3} g_{4} T=1$. Thus $\mathcal{H} / \Gamma_{2,2,2,2}(a, b, c, d)$ has signature $\{0,\{2,2,2,2\}, 1\}$.

3.4. Comparison with $\Gamma_{0}^{*}(q)$. One can check that $\Gamma_{2,2,2}(v, 0)=\Gamma_{0}^{*}(v)$ for $v=5$, 6 , or 8 , and

$$
\Gamma_{0}^{*}(9)=\left(\begin{array}{cc}
1 & \frac{1}{6} \\
0 & 1
\end{array}\right) \Gamma_{2,2,2}\left(9, \frac{1}{6}\right)\left(\begin{array}{cc}
1 & \frac{1}{6} \\
0 & 1
\end{array}\right)^{-1} .
$$

So $\Gamma_{2,2,2}$ gives the desired deformation of these groups.

Note that there are various symmetries to $\Gamma_{2,2,2}$ and the same group can appear several times. For example, $\Gamma_{0}^{*}(5)$ appears as $\Gamma_{2,2,2}(5,0), \Gamma_{2,2,2}(20,0)$ and $\Gamma_{2,2,2}\left(5, \frac{1}{5}\right)$. More generally, $\Gamma_{2,2,2}(a, 0)$ is isomorphic to $\Gamma_{2,2,2}(4 a /(4-a), 0)$. Also, $\Gamma_{2,2,2}(a, b)=\Gamma_{2,2,2}(a,-b)$. Other symmetries will be visible in our plots in Section 4.

Also, $\Gamma_{2,2,2,2}(-1 / 3,0,1 / 3,1 /(2 \sqrt{11}))=\Gamma_{0}^{*}(11)$. So $\Gamma_{2,2,2,2}$ will be our deformation of $\Gamma_{0}^{*}(11)$.

In the next section we use the generators given above in our numerical calculations.

\section{Tracking the MaAss Forms}

Let $\Gamma$ be one of the groups described in the previous section. We use the methods described in [FJ] to locate Maass forms on those groups. Those methods are summarized in the next section. Once one Maass form is located, we search for a small deformation of the group such that there is a Maass form with an eigenvalue close to that of the original Maass form. We then use those two values to interpolate or extrapolate to find a starting point for locating more deformations of the original form. The end result is a sequence of closely spaced deformations for which we have Maass forms with slowly changing eigenvalue and Fourier coefficients. The great accuracy to which we find the individual forms, coupled with the small changes to the Maass form as we vary the group, provides persuasive evidence that we are indeed tracking the deformation of a Maass form.

4.1 Locating Maass forms. The following is a summary of the methods used to locate an individual Maass form. Given generators $\left\{g_{j}\right\}$ of $\Gamma$, we produce an overdetermined system of linear equations which uses a truncation of the Fourier expansion of $f$

$$
\tilde{f}(z)=\sqrt{y} \sum_{|n| \leq M, n \neq 0} a_{n} K_{i R}(2 \pi|n| y) \exp (2 \pi i n x),
$$

where the $a_{i}$ are complex unknowns. Note that we assume $a_{0}=0$ (which excludes all Eisenstein series when we have only one cusp) and we also normalize one of the coefficients (usually $a_{1}$ ) to equal 1 . Also note that this of course depends on $R$ (or equivalently on $\lambda$ ). For most of the examples in this paper, we choose $M$ so that the error caused by the truncation is around $10^{-8}$ for points in the fundamental domain of $\Gamma$.

We treat the $\left\{a_{n}\right\}$ as $4 M-2$ real unknowns. Next we choose $N$ points $z_{i}$ (where $N>4 M$ ) on a horizontal line in $\mathcal{H}$. These points are mapped by the generators to 
points $g_{j} z_{i}=z_{i}^{*}$ higher up in $\mathcal{H}$. If $f$ is a Maass form on $\mathcal{H} / \Gamma$, then $f\left(z_{i}\right)=f\left(z_{i}^{*}\right)$ (or more generally $f\left(z_{i}\right)=\chi\left(g_{j}\right) f\left(z_{i}^{*}\right)$ where $\chi$ is a character). The $N$ equations

$$
\tilde{f}\left(z_{i}\right)=\tilde{f}\left(z_{i}^{*}\right)
$$

constitute an overdetermined system $A x=b$ in $4 M-2$ unknowns. If $R$ is an eigenvalue of a Maass form on $\Gamma$, then this system should be consistent to within the error caused by the truncation.

Next we determine the least square solution $\tilde{x}$ (using $Q R$-factorization) to this system of equations. We then use the norm of the error, $\|A \tilde{x}-b\|_{2}$, as a measure of how close $\lambda$ is to an eigenvalue. If $\lambda$ is really an eigenvalue, then $\|A \tilde{x}-b\|_{2}$ should be roughly the size of the truncation error. In our initial calculations in cases where earlier data were available we found this to be true. We also found that away from eigenvalues (i.e., if we choose $R$ randomly) the error is generally of size 1 , independent of the size of the truncation error. We take $\|A \tilde{x}-b\|_{2}$ to be a measure of distance between $R$ and a "true" eigenvalue for $\Gamma$, and we have found this measure to vary smoothly and to be consistent with various other checks, which we describe below. Thus, it seems reasonable to say that these functions are a factor of $10^{8}$ closer to being invariant under $\Gamma$ than a randomly chosen function.

There are a number of error checks. If $\Gamma$ is arithmetic, then the Fourier coefficients will be multiplicative, and we find that the above method produces functions whose coefficients are multiplicative to better than the truncation error. These can be viewed as independent 1 in $10^{8}$ error checks, which render it very likely that the functions produced are indeed Maass forms. In other words, the possibility of a "false alarm" is extremely small, and we have high confidence that the program is finding the Maass forms for $\Gamma$.

For nonarithmetic groups there are no Hecke relations, but there are other persuasive checks. We start with a general Fourier expansion with complex coefficients. For the Maass forms we find, the functions are real to very high accuracy (to an even higher accuracy than the truncation error). In general, when we are far from an eigenvalue, the system of equations is far from consistent and the approximate solutions are far from real.

A final check is the size of the Fourier coefficients. For arithmetic $\Gamma$ all the coefficients we have found fit the Ramanujan-Peterson conjecture $\left|a_{p}\right|<2$. For nonarithmetic groups that bound is not true in general, but it is still conjectured that $a_{n} \ll n^{\epsilon}$. Also, we do in fact find that if $R$ is close to an eigenvalue, then the $a_{n}$ from the least-squares solution are much smaller (and not growing as a function of $n$ ) than those from random $R$.

Having identified one Maass form, it can serve as a starting point for locating Maass forms on nearby groups. If no Maass forms are detected on nearby groups, then we can state with confidence that the Maass form is destroyed by all deformations. If a Maass form is detected, then by finding a succession of nearby Maass forms on nearby groups, we can state with confidence that the Maass form survives as the group is deformed. We do indeed find continuous families of Maass forms in all cases which we checked, so we suggest that on all groups which admit deformations, each Maass form has a continuous family of deformations on which it lives.

The following sections illustrate some of the deformations associated with Maass forms on $\Gamma_{2,2,2}$. Since the deformation space of this group is 2 (real) dimensional, it is possible to visualize the deformations of the Maass forms. In the case of 
$\Gamma_{2,2,2,2}$ there is a 4-dimensional deformation space, and we must be less direct in our demonstration that the Maass forms live on a 3-dimensional subspace.

Samples of our data can be found at http://www.math.chalmers.se/ sj/Maass/.

4.1.1. Are we really finding cusp forms? One important issue is justifying that we are actually finding cusp forms, as opposed to the residue of a pole of an Eisenstein series. By construction our functions vanish at the cusps (because $a_{0}=0$ and $\Gamma$ has only one cusp). But in the nonarithmetic cases it is possible that we are actually finding a resonance with extremely small constant term: smaller than the truncation error. This would be a serious concern if we only considered a few examples and if those involved very small deformations of an arithmetic group. But, as can be seen below, we consider deformations which are quite far from an arithmetic group, and we consistently find our error check to be a factor of $10^{8}$ smaller than expected for random data. So, unless the Maass forms deform in a way such that the constant term always stays extremely small (a factor of $10^{8}$ smaller than the other coefficients), then the functions we find are cusp forms.

We have also done calculations where we include a (nonzero) constant term

$$
\sqrt{y}\left(a_{0} \cdot \cos R \ln y+b_{0} \cdot \sin R \ln y\right) .
$$

We know that there is a 1-dimensional space of Eisenstein series, since we have exactly one cusp. Numerically we find a unique solution at a generic point in our deformation space. This solution does not vary (more than the truncation error) when we vary the chosen points $z_{i}$. This solution should be the Eisenstein series. At a point where we claim that we find cups forms, these calculations give different solutions for each set of points $z_{i}$. Taking a suitable linear combination of any two of these solutions gives a solution with constant coefficients $a_{0}$ and $b_{0}$ less than the truncation error. This solution (of course) agrees with the one we get when forcing the constant coefficients to be zero. Setting $a_{0}=10^{-5}$ and $b_{0}=0$ gave errors consistently about 100 times larger than when they were both set to zero. We also remark that we normalized the size of the terms in such a way that the coefficients in the matrix $A$ were of size 1 both for the constant coefficients and the first nonconstant coefficients.

It would be interesting to input our deformations into the method of Avelin [A] to track the motion of the zeros and poles of the scattering matrix. See comment (1) in Section 4.6 for a particularly interesting example.

If the cusp forms are becoming resonances with uniformly very small constant term, then it would be interesting to find an explanation for this phenomenon.

4.2. Tracking the eigenvalues. Our first example is an exhaustive search for all Maass forms for $\Gamma_{2,2,2}(a, b)$ in the box

$$
(5 \leq a \leq 6) \times(0 \leq b \leq 0.16) \times(11 \leq R \leq 12) .
$$

Note that $(a, b)=(5,0)$ corresponds to $\Gamma_{0}^{*}(5)$ and $(a, b)=(6,0)$ corresponds to $\Gamma_{0}^{*}(6)$.

When $b=0$, the group has an extra symmetry, as can be seen in Figure 3.1. In this case the Maass forms are classified as "even" or "odd" according to whether they are even or odd functions of $x$. 

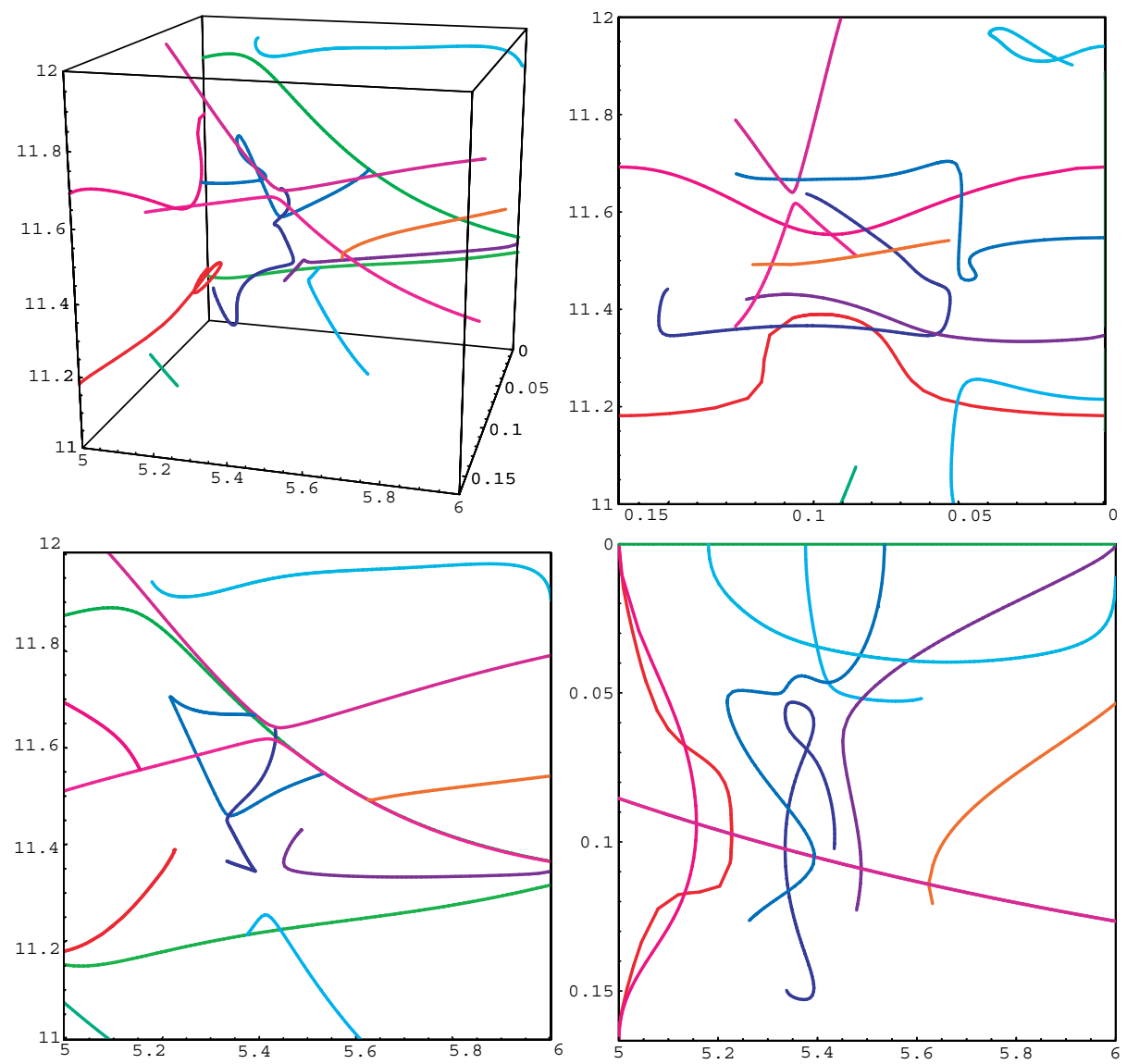

FIgURE 4.1. Maass forms on deformations of $\Gamma_{0}^{*}(5)$.

The plots in Figure 4.1 depict the thirteen equivalence classes of Maass forms which intersect the region of the search. The cube in the upper left shows all of the forms. The left/right, front/back, and up/down axes correspond to $a, b$, and $R$, respectively. The back-left edge corresponds to $\Gamma_{0}^{*}(5)$, and the back-right edge corresponds to $\Gamma_{0}^{*}(6)$. The back face, where $b=0$, corresponds to those deformations having the extra symmetry described above.

Odd Maass forms on $\Gamma_{0}(N)$ survive under deformations preserving the left-right symmetry of the fundamental domain because the Eisenstein series are even and so it is not possible for the odd forms to dissolve into the continuous spectrum. This is illustrated in the diagram by the two equivalence classes contained in the back face of the cube. Thus, the figure shows that there are four Maass forms on $\Gamma_{0}^{*}(5)$ with eigenvalue $11 \leq R \leq 12$, with two of them odd and two of them even.

Note: A separate search found that there were indeed exactly four Maass forms in that range for $\Gamma_{0}^{*}(5)$. Also, there are even Maass forms for $\Gamma_{0}^{*}(6)$ with $11 \leq R \leq 12$, but for small deformations they leave the search region.

Proceeding counterclockwise from the cube, the other figures show the projections onto the back, bottom, and left faces of the cube, respectively. 
The view from the top shows an extra symmetry along the curve from $(a, b)=$ $(5,0.0854)$ to $(a, b)=(6,0.1266)$. We did not trace all of the Maass forms past their point of symmetry.

Further observations from these figures are made in Section 4.5.

4.3. Nonarithmetic examples. We know that Maass forms exist on congruence groups, and the deformations of these arithmetic Maass forms give Maass forms on nonarithmetic groups. It is natural to ask whether all Maass forms can be "explained" by their existence on arithmetic groups. That is, are all Maass forms a deformation of a Maass form on an arithmetic group? We give four examples below which show that, unfortunately, the answer seems to be "no".

Each of the curves in Figure 4.2 begins and ends on equivalent groups, so they correspond to closed paths in Teichmüller space. We believe that none of the points on those curves correspond to arithmetic groups. (We have not been able to verify this directly, but we have checked that none of those Maass forms have multiplicative coefficients.)
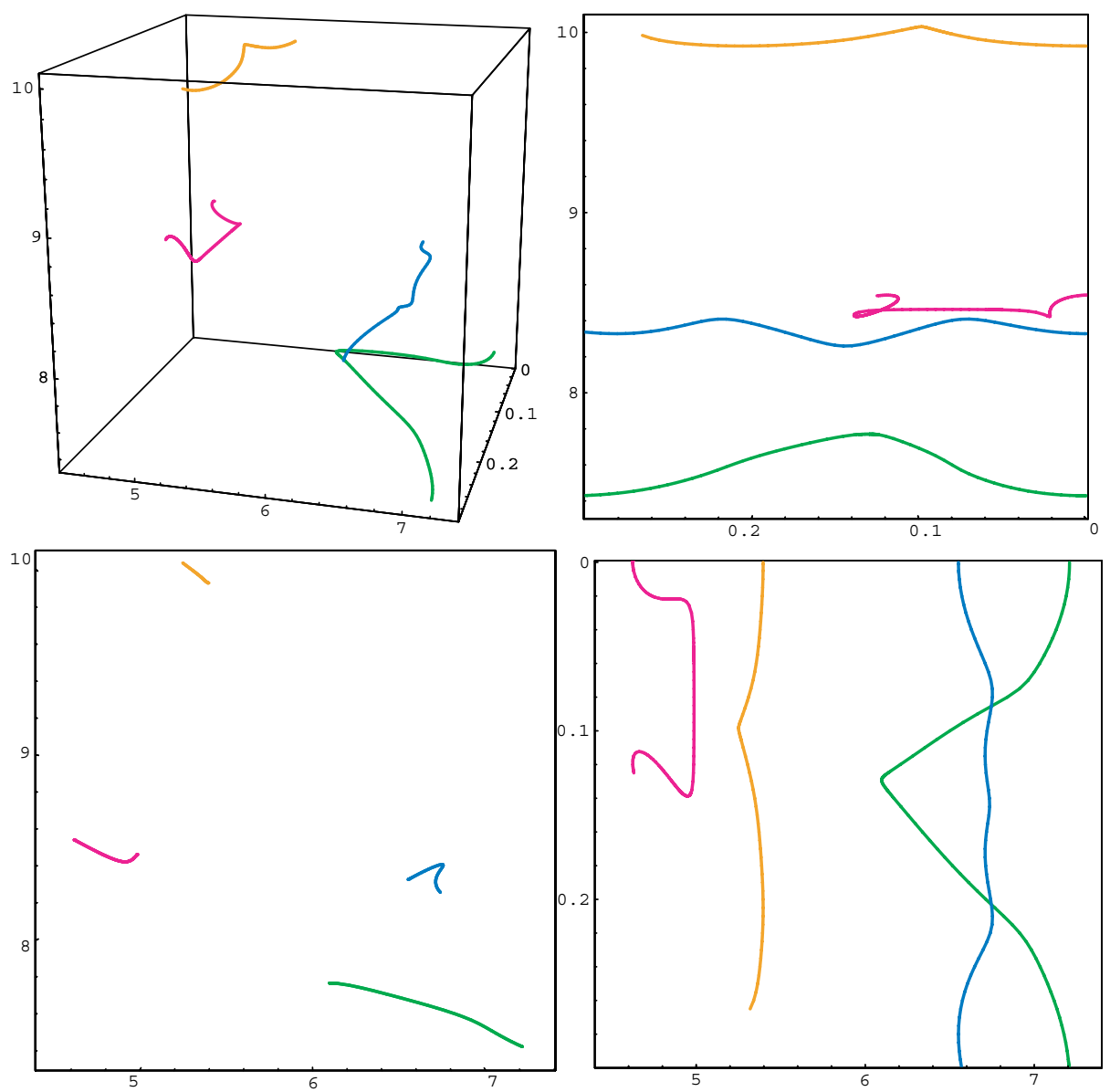

Figure 4.2. Maass forms which do not arise from an arithmetic group 


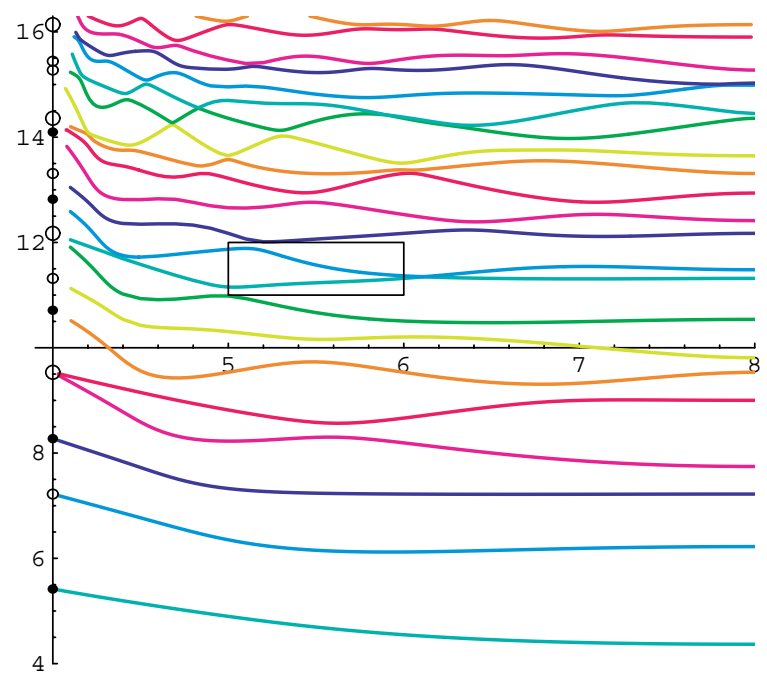

Figure 4.3. Odd Maass forms in the $b=0$ plane, for $4<a \leq 8$ and $0 \leq R \leq 16.3$. The black dots are odd eigenvalues for newforms on $\Gamma_{0}^{*}(4)$, the small circles are odd newforms on $\Gamma_{0}^{*}(2)$, the large circles are odd eigenvalues on $\Gamma(1)$, and the rectangle is the back face of the cube in Figure 4.1

4.4. Level dynamics of odd forms. The odd Maass forms on $\Gamma_{0}(5)$ or $\Gamma_{0}(6)$ can be deformed in the $b=0$ plane, which makes their behavior under deformation somewhat easier to visualize. Figure 4.3 shows the deformations of the odd Maass forms on $\Gamma_{0}^{*}(5)$ for $0<R<16.3$. The rectangle in the figure corresponds to the back face of the cube in Figure 4.1. For $a>8$ the deformation repeats according to the rule $a \rightarrow 4 a /(a-4)$. The limit $a \rightarrow 4$ corresponds to the merging of two cusps (and so $a=4$ can never be reached). Nevertheless, $\Gamma_{2,2,2}(4,0)$ can be interpreted as $\Gamma_{0}^{*}(4)$, and as $a \rightarrow 4$, the eigenvalues approach those on $\Gamma_{0}^{*}(4)$.

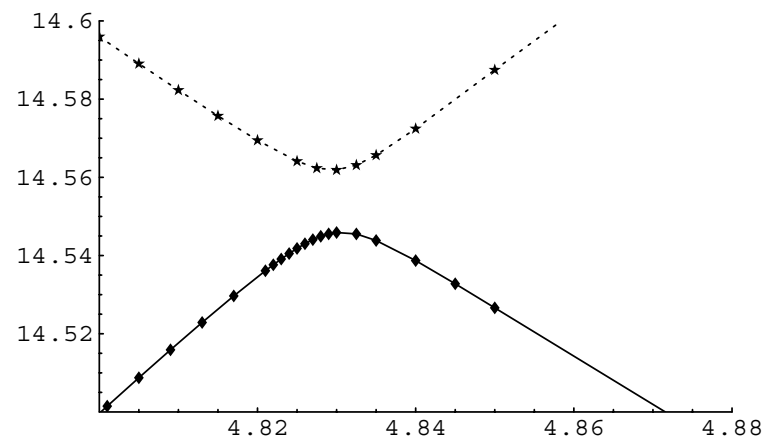

Figure 4.4. A closeup of the avoided crossing near $(a, R)=$ $(4.83,14.55)$. The dots show the points calculated by the computer program. 
TABLE 4.1 .

\begin{tabular}{|l|c|}
\hline \multicolumn{1}{|c|}{$(a, b, c, d)$} & $R$ \\
\hline$(-0.3333333,0,0.3333333,0.1507556)$ & 11.8005163 \\
$(-0.31,0.03,0.37,0.1406783)$ & 11.8076532 \\
$(-0.31,0.03,0.3704,0.1404517)$ & 11.8092074 \\
$(-0.31,0.03,0.37015,0.1405936)$ & 11.8082495 \\
$(-0.31,0.03,0.3711848,0.14)$ & 11.8120386 \\
$(-0.3121659,0.03,0.37,0.14)$ & 11.8172635 \\
\hline
\end{tabular}

Figure 4.1 also shows the phenomenon of "avoided crossing", a manifestation of the fact that a multiplicity in the spectrum is a codimension 2 condition. A closeup of the avoided crossing near $(a, R)=(4.83,14.55)$ is given in Figure 4.4. In a future paper we will include a more detailed study of the level dynamics of the odd Maass forms.

4.5. Deformations of $\Gamma_{2,2,2,2}$. Since the Teichmüller space of $\Gamma_{0}(11)$ is 4-dimensional, we are not able to draw pictures like those above. Our argument that the Maass forms live on a 3 -dimensional set is as follows.

Suppose $f$ is a Maass form on $\Gamma_{2,2,2,2}\left(a_{0}, b_{0}, c_{0}, d_{0}\right)$ with eigenvalue $R_{0}$. Now suppose we change three of $a_{0}, b_{0}, c_{0}, d_{0}$ very slightly. If the Maass form lives on a 3 -dimensional space, we will be able to change $R_{0}$ and the other parameter slightly and find another Maass form. Suppose we make several choices for three of the parameters (and change them in a slightly different way each time), and in each case we can slightly adjust $R_{0}$ and the other parameter and again find a Maass form. Then that is persuasive evidence that the Maass form lives on a 3-dimensional set.

Table 4.1 is a representative example of these calculations. The top line is the data for an odd Maass form of $\Gamma_{0}(11)$, which corresponds to $(a, b, c, d)=$ $\left(-\frac{1}{3}, 0, \frac{1}{3}, \frac{1}{2 \sqrt{11}}\right)$. The other entries are deformations of that Maass form, where in each case the values given to five or fewer decimal places were chosen exactly, and the values given to seven decimal places are approximations determined in the search.

4.6. Additional observations. Here we address various observations which have not been discussed previously.

(1) It was noted that odd Maass forms on $\Gamma_{2,2,2}(a, b)$ live on the plane of deformations with $b=0$. Figures 4.1 and 4.2 strongly suggest that even Maass forms on $\Gamma_{2,2,2}(a, 0)$ live on a set which meets the $b=0$ plane perpendicularly. This is confirmed by Avelin's A observation that under deformations of the form $(a, b)=(5, t)$, the zeros of the scattering term $\varphi(s)$ have 4 th order contact with the $\Re(s)=\frac{1}{2}$ axis. The 4 th order contact indicates that the deformation is in the (unique) direction in which the Phillips-Sarnak condition for destruction is not satisfied. As a specific example, consider the eigenvalue $R_{0} \approx 5.436180461$ for $\Gamma_{0}(5)$. Fitting to our data, we find that the initial path of the deformation is along the curve $a \approx 5+66.729 b^{2}-2.1 \times 10^{4} b^{4}$ with $R \approx R_{0}-25.429 b^{2}+8.3 \times 10^{3} b^{4}$. 
Deformation along the path $(a, b)=(5,0)+t(0,1)+t^{2}(66.729,0)$ should find that the zero of the scattering matrix has 6th order contact with the $\Re(s)=\frac{1}{2}$ axis. Using the next term (assuming our value of $-2.1 \times 10^{4}$ is sufficiently accurate) should give eighth order contact.

(2) There are Maass forms on $\Gamma_{2,2,2}(a, b)$ which can be deformed in two different directions. For example, consider the points $(5.53487,0,11.54704)$ and $(5.120,0.0919,11.9671)$ in Figure 4.1] The diagrams show two 1-parameter deformations intersecting at each of those points. However, there is only one, not two, Maass form at the intersection point. That is, those points correspond to a single Maass form which can be deformed in two independent directions, as opposed to an eigenvalue of multiplicity two. (We ruled out the possibility of a multiple eigenvalue by verifying that there were no Maass forms whose first Fourier coefficient vanished. We also checked that as you approach the point along either path, the coefficients have the same limiting value.)

Note that the Maass form at $(5.53487,0,11.54704)$ is odd, so it has the expected deformation in the $b=0$ plane. However, it also can be deformed along a path which initially is perpendicular to the $b=0$ plane.

(3) There are two places visible in Figure 4.1 where the fundamental domain of the group $\Gamma_{2,2,2}(a, b)$ has an extra symmetry which permits us to distinguish between "even" and "odd" forms. One such place is the plane $b=0$, which is the back face of the cube in the diagrams. Another place lies along the curve from $(a, b)=(5,0.0854)$ to $(a, b)=(6,0.1266)$, which is clearly visible in Figure 4.1. Since the Eisenstein series are even, the odd Maass forms have deformations which lie in those regions, which indeed can be seen in the figure.

As mentioned in the previous comment, the remaining Maass forms appear to cross those lines of symmetry perpendicularly. Furthermore, those remaining curves either intersect an arithmetic group or they intersect the path of an odd Maass form as they cross one of the lines of symmetry. So, in some sense, all of these Maass forms have some connection with an arithmetic group.

(4) The calculations we present here are illuminating, but the main features are not unexpected. In particular, one should expect that Maass forms live on $(d-1)$-dimensional real analytic subvarieties of Teichmüller space. As described in Section 1.3, for each cusp form Phillips and Sarnak define a map from Teichmüller space to the half-plane $\Re(s) \leq \frac{1}{2}$, such that a cusp form is not destroyed if the point lies on the line $\Re(s)=\frac{1}{2}$. One expects this map to be real analytic, and one might also expect it to be nondegenerate, which our calculations appear to confirm. Thus, the curves in Figures 4.1 and 4.2 are the inverse image of the line $\Re(s)=\frac{1}{2}$. It is unclear whether it is surprising that those curves have multiple points

Note that the above paragraph concerns the variety on which the Phillips and Sarnak integral vanishes, as opposed to the variety on which the deformed cusp form lives. However, both varieties have (real) codimension 1, suggesting that they are the same. It is not clear how this relates to Avelin's A observation of scattering zeros having 4th order contact with the $\Re(s)=\frac{1}{2}$ axis. 
Sarnak has suggested to us that the dissolving condition for the deformation of a Maass form can be used to write down a differential equation which is satisfied by the curves we have found. It would be interesting to write down such an equation.

\section{Questions}

(1) Do the calculations in this paper actually find deformed cusp forms or merely Eisenstein series with uniformly very small constant term? In the latter case, why do the constant terms stay so small?

The remaining questions assume that our calculations actually find cusp forms.

(2) Is there a Weyl law for equivalence classes of Maass forms?

(3) Can a Maass form ever be deformed to give a different Maass form on the same group? Does the answer change if one restricts to smooth deformations (so, for example, odd Maass forms in the examples above can never leave the plane $b=0)$ ?

(4) In the case of $\Gamma_{2,2,2}(a, b)$, or any other group with a 2 real parameter deformation space, how can one detect Maass forms which can be deformed in two independent directions? Do those Maass forms have any special properties?

(5) Is it surprising that there exist Maass forms which cannot be smoothly deformed to give a Maass form on an arithmetic group? It is possible that such forms always arise as the deformation of an "odd" Maass form?

\section{REFERENCES}

[A] H. Avelin, Research Announcement on the deformation of cusp forms, U.U.D.M. Report 2002:26, Uppsala Univ.

[Co] H. Cohn, A numerical survey of the reduction of modular curve genus by Fricke's involutions, Number theory (New York, 1989/1990), 85-104, Springer, New York, 1991. MR1124636 (92f:11060)

[C1] Y. Colin de Verdiere, Pseudo-Laplacians I, Ann. Inst. Fourier 32 (1983), 275-286. MR0688031 (84k:58221)

[C2] Y. Colin de Verdiere, Pseudo-Laplacians II, Ann. Inst. Fourier 33 (1983), 87-113. MR.0699488 (84k:58222)

[FJ] D. Farmer and S. Lemurell, in preparation.

[H1] D. Hejhal, Eigenvalues of the Laplacian for Hecke triangle groups, Mem. Amer. Math. Soc. (1992), no. 469, 165 pp. MR1106989 (93f:11043)

[H2] D. Hejhal, On Eigenfunctions of the Laplacian for Hecke triangle groups, in Emerging applications of number theory, Springer, 1999, 291-315. MR1691537 (2000f:11063)

[H3] D. Hejhal and S. Arno, On Fourier coefficients of Maass waveforms for $\operatorname{PSL}(2, Z)$, Math. Comp. 61 (1993), 245-267 and S11-S16. MR1199991 (94a:11062)

[Iw] H. Iwaniec, An introduction to the spectral theory of automorphic forms, Bibl. Rev. Mat. Iber., Madrid, 1995 (Reprinted by AMS). MR.1325466 (96f:11078)

[L] W. Luo, Nonvanishing of $L$-values and the Weyl law, Ann. of Math. (2), 154 (2001) no. 2, 477-502. MR.1865978 (2002i:11084)

[P] Y. Petridis, Perturbation of scattering poles for hyperbolic surfaces and central values of $L$-series, Duke Math. J. 103, no. 1 (2000), 101-130. MR.1758241 (2001d:11057)

[PS1] R. Phillips and P. Sarnak, On cusp forms for cofinite subgroups of $S L(2, R)$, Invent. Math. 80 (1985), 339-364. MR0788414 (86m:11037)

[PS2] R. Phillips and P. Sarnak, The Weyl theorem and the deformation of discrete groups, Comm. pure and applied math. 38 (1985), 853-866. MR0812352 (87f:11035)

[PS3] R. Phillips and P. Sarnak, Perturbation theory for the Laplacian on automorphic functions, J. Amer. Math. Soc. 5 (1992), 1-32. MR1127079 (92g:11056) 
[S1] P. Sarnak, On cusp forms, The Selberg trace formula and related topics (Brunswick, Maine, 1984), 393-407, Contemp. Math., 53, Amer. Math. Soc., Providence, RI, 1986. MR0853570 (87j:11047)

[S2] P. Sarnak, On cusp forms. II. Festschrift in honor of I. I. Piatetski-Shapiro on the occasion of his sixtieth birthday, Part II (Ramat Aviv, 1989), 237-250, Israel Math. Conf. Proc., 3, Weizmann, Jerusalem, 1990. MR.1159118 (93e:11068)

[Ta] K. Takeuchi, A characterization of arithmetic Fuchsian groups, J. Math. Soc. Japan, 27 (1975), 600-612. MR0398991 (53:2842)

[W] S. Wolpert, Disappearance of cusp forms in special families, Ann. of Math. (2) 139 (1994), no. 2, 239-291. MR $1274093(95 \mathrm{e}: 11062)$

American Institute of Mathematics, 360 Portage Avenue, Palo Alto, California 94307

E-mail address: farmer@aimath.org

Chalmers University of Technology, SE-412 96 Göteborg, Sweden

E-mail address: sj@math.chalmers.se 